\title{
Flat Finned Tube Heat Exchanger Outside the Numerical Simulation of Fow and Heat Transfer Characteristics
}

\author{
Wen Cao ${ }^{a}$, Qiang Li ${ }^{b}$, Zhigang Tian ${ }^{c}$ \\ Vehicle Application Department, Changchun Institute of Engineering and Technology, Changchun, \\ Economic Development Zone, China \\ a58986717@qq.com, ${ }^{b}$ liqiang33@126.com, ${ }^{c}$ tian5333@126.com
}

Keywords: Flat fin; Heat exchanger; Numerical simulation; Field synergy principle.

\begin{abstract}
This paper, by using CFD software FLUENT to vehicle flat fin heat exchanger fin surface fluid flow and heat transfer process in the numerical simulation of the internal flow field, temperature field and heat exchanger heat exchanger in the import and export pressure drop and the average convective heat transfer coefficient of finned surface, etc. According to the simulation results, the fin surface convective heat transfer coefficient increases with the increase of wind speed, but the increase rate gradually decreases; Heat exchanger in the import and export pressure drop increases with the increase of wind speed, and increases its speed was accelerated. Convection heat transfer, using the field synergy principle further analysis found that the velocity increases with increase in heat is the root cause of the increase of air volume; Increased speed cause the average field synergy Angle increase in the convective heat transfer process, make the velocity field and temperature field of collaborative variation.
\end{abstract}

\section{Introduction}

With the continuous development and progress of computer technology, the computing speed of central processing unit (CPU) is greatly improved. Furthermore, the use of computational fluid dynamics (CFD) technology to simulate various practical problems has been rapidly developed. Using CFD technology to simulate practical problems has the advantages of low cost, fast speed, and being able to simulate complex process changes. In particular, it has unique advantages for some problems that are difficult to measure or have large experimental investment in the experimental process. More and more attention has been paid to the study of CFD in fluid mechanics and heat transfer. In this paper, CFD software FLUENT is used to simulate the air flow and heat transfer process on the surface of the flat fin of the vehicle tubular fin heat exchanger, and the flow characteristics of the air in the heat exchanger as well as the convection heat transfer process between the air and the fin are studied. At the same time, the process of convection heat transfer is further analyzed by applying the field cooperation principle.

\subsection{Physical model description}

The relevant structural parameters of the flat-fin heat exchanger of the vehicle are shown in figure 
1. The thickness of the aluminum fin is $0.11 \mathrm{~mm}$, the outer diameter of the inner copper pipe is $9.52 \mathrm{~mm}$, the spacing between pipes is $21.7 \mathrm{~mm}$, and the spacing between rows is $25 \mathrm{~mm}$. The calculation area is taken between the two fins, and the middle part is divided into the fluid area. The thickness of the two fins is generally the boundary, and the periodic boundary conditions are given. In order to reduce the air inlet effect and outlet reflux, the pipe diameter of the inlet and outlet is extended about 5 times (FIG. 1 is the parameter of the flat fin after the extension of the inlet and outlet).

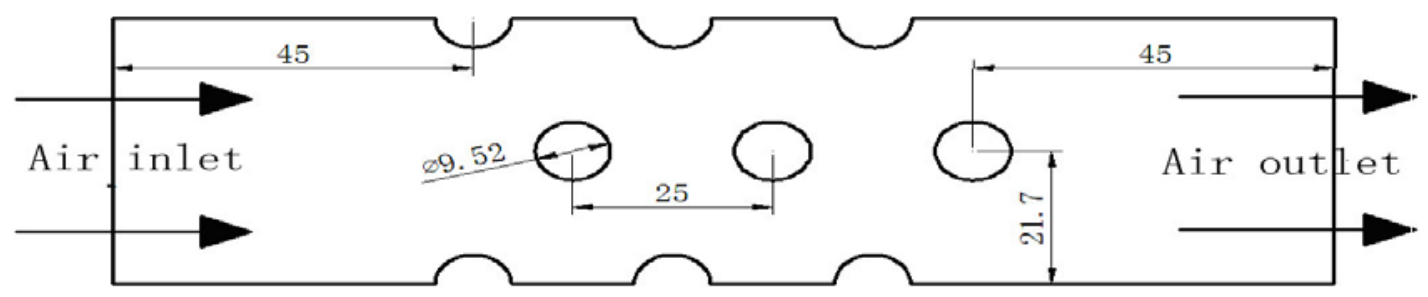

Fig. 1. Fin dimensions of heat exchanger

\section{Mathematical model and its solution}

\subsection{Mathematical model}

For the above physical model, the heat transfer process is simplified as follows:

(1) the physical parameters of the fluid change little with the air temperature, and are approximately constant;

(2) the fluid does not slip on the wall surface;

(3) the flow is steady and symmetrical;

(4) the impact of natural convection and radiation heat transfer is not considered;

(5) ignoring the thermal contact resistance between the copper tube and the fin;

(6) since the fin thickness is far less than the fin height, it is considered that the heat transfer on the three sides of the fin thickness is zero.

Based on the above assumptions, the mathematical model describing the problem is obtained, and the main control equations are listed as follows:

Continuity equation:

$$
\frac{\partial}{\partial x_{i}}\left(\rho u_{i}\right)=0
$$

Momentum equation:

$$
\frac{\partial\left(\rho u_{i} u_{j}\right)}{\partial x_{j}}=-\frac{\partial p}{\partial x_{i}}+\frac{\partial}{\partial x_{j}}\left(\mu \frac{\partial u_{i}}{\partial x_{j}}-\rho u_{i} u_{j}\right)
$$

Air side energy equation:

$$
\frac{\partial}{\partial x_{i}}\left[u_{i}(\rho E+P)\right]=\frac{\partial}{\partial x_{i}}\left(\lambda_{\text {eff }} \frac{\partial T}{\partial x_{i}}\right)
$$

The heat transfer equation in the solid region: 


$$
\frac{\partial}{\partial x_{i}}\left(\lambda_{s} \frac{\partial T}{\partial x_{i}}\right)=0
$$

Use the standard $k_{-} \varepsilon$ model:

$k$ equation:

$$
u_{j} \frac{\partial k}{\partial x_{j}}=\frac{\partial}{\partial x_{j}}\left[\left(\mu+\frac{\mu_{t}}{\sigma_{k}}\right) \frac{\partial k}{\partial x_{j}}\right]+G_{k}-\rho \varepsilon
$$

$\varepsilon$ equation:

$$
u_{j} \frac{\partial \varepsilon}{\partial x_{j}}=\frac{\partial}{\partial x_{j}}\left[\left(\mu+\frac{\mu_{t}}{\sigma_{\varepsilon}}\right) \frac{\partial \varepsilon}{\partial x_{j}}\right]+C_{1} \frac{\varepsilon}{k} G_{k}-C_{2} \rho \frac{\varepsilon^{2}}{k}
$$

All the subscripts above satisfy Einstein's summation convention. Type, ${ }_{i}$ as the air in $x_{i}$ velocity of direction, $\mathrm{m} / \mathrm{s} ; \quad \rho$ is gas density, $\mathrm{kg} / \mathrm{m} 3$; $\mathrm{P}$ Is the static pressure of the gas, $\mathrm{Pa}$; $\mathrm{E}$ is the specific internal energy of gas, $\mathrm{J} / \mathrm{kg} ; \lambda_{\text {eff }}=\lambda+\lambda_{f}$ is the effective heat transfer coefficient, $\lambda$ is the heat transfer coefficient of laminar flow, $\lambda_{f}$ Is the heat transfer coefficient of turbulence, $\left.W /\left(m^{2} \cdot k\right)\right) ; \quad \mu_{t}=\rho C_{\mu} \frac{k^{2}}{\varepsilon}, \quad G_{k}=\mu_{t}\left(\frac{\partial u_{i}}{\partial x_{j}}+\frac{\partial u_{j}}{\partial x_{i}}\right) \frac{\partial u_{i}}{\partial x_{j}} \quad$ Is the turbulent kinetic energy generated due to the average velocity gradient, $\mathrm{J} ; \sigma_{k}$ as for the $k$ the turbulence prandtl number, $\sigma_{\varepsilon}$ as for the $\varepsilon$ The turbulence prandtl number. The above standard $k_{-} \varepsilon$ the values of equation model parameters are shown in table 1.

Table 1 standard $k_{-} \varepsilon$ model parameters

\begin{tabular}{ccccc}
\hline$C_{\mu}$ & $C_{1}$ & $C_{2}$ & $\sigma_{k}$ & $\sigma_{\varepsilon}$ \\
\hline 0.09 & 1.44 & 1.92 & 1.0 & 1.3 \\
\hline
\end{tabular}

Determination of boundary conditions:

The air inlet boundary is given inlet wind speed and temperature;

Set the relative pressure at the outlet boundary condition to zero.

The setting of boundary conditions on fluid-solid coupling surfaces is determined according to the wall function method [1].

\subsection{Solution ideas and methods}

The CFD fluid-solid conjugate heat transfer technique is used to calculate the flow and heat transfer. Choose the standard $k_{-} \varepsilon$ model, In the calculation process, finite volume method is adopted to discretize the equation. The momentum equation adopts the first-order windward format, and the energy equation adopts the second-order windward format. Meanwhile, mass, momentum and $k, \varepsilon$ two equations and the energy equation, calculate the control energy equation residual difference to 10-6. SIMPLE algorithm is adopted to solve the control equation [1].

By changing the velocity of inlet air, the average convective heat transfer coefficient and pressure drop of inlet and outlet of air and fin were calculated. Import air temperature at $35{ }^{\circ} \mathrm{C}$, tube fluid for 
$9{ }^{\circ} \mathrm{C}$, wind speed: $0.6 \mathrm{~m} / \mathrm{s}, 1.2 \mathrm{~m} / \mathrm{s}, 1.8 \mathrm{~m} / \mathrm{s}, 2.4 \mathrm{~m} / \mathrm{s}, 3.0 \mathrm{~m} / \mathrm{s}, 4.0 \mathrm{~m} / \mathrm{s}, 5.0 \mathrm{~m} / \mathrm{s}, 6.0 \mathrm{~m} / \mathrm{s}$, a total of eight set of conditions.

\section{Analysis of solution results}

\subsection{Model results analysis}

The distribution of air flow field, temperature field and fin surface temperature can be obtained by simulating the eight different wind speeds. The calculation results of three representative working conditions were selected from eight working conditions for analysis. FIG. 2, FIG. 3 and FIG.4 respectively show the temperature field and velocity field of air under wind speed of $0.6 \mathrm{~m} / \mathrm{s}, 2.4 \mathrm{~m} / \mathrm{s}$ and $5.0 \mathrm{~m} / \mathrm{s}$. In the figure, temperature and velocity values of some local areas are standardized.

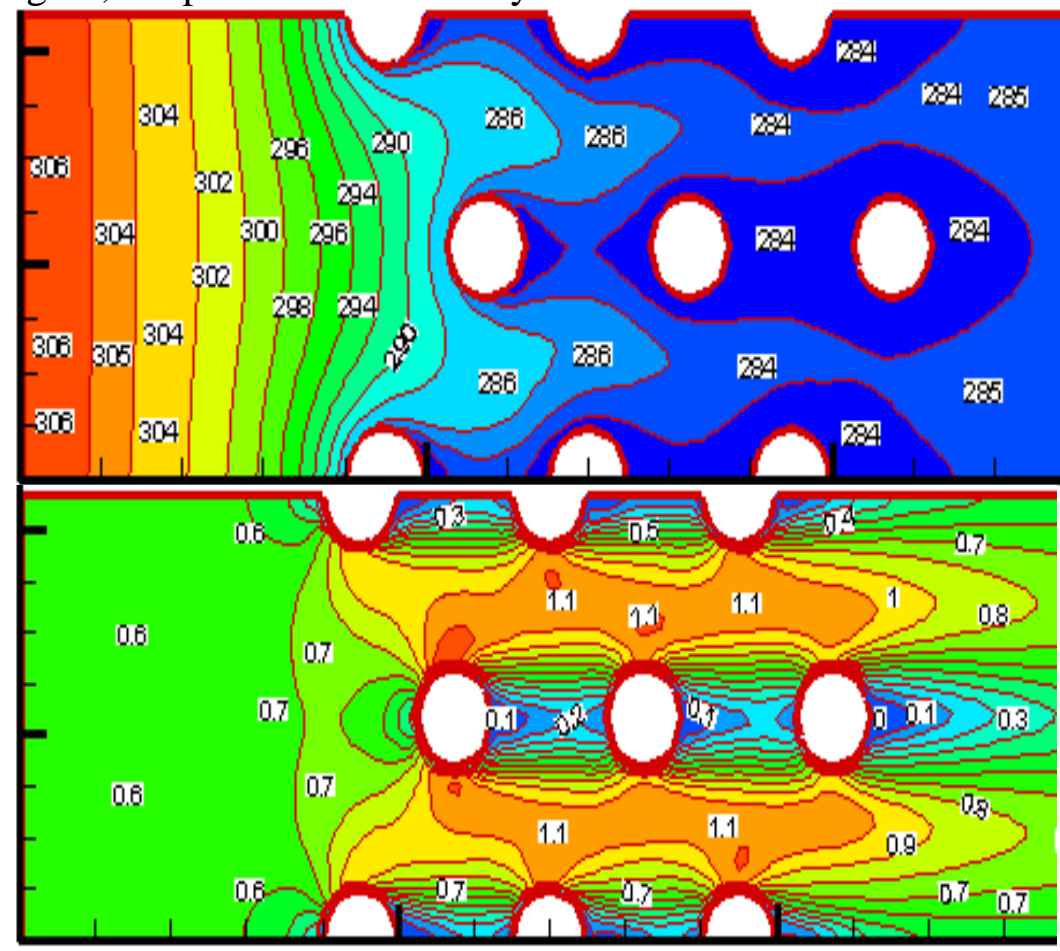

Fig. 2 cloud diagram of air temperature and velocity distribution when $\mathrm{V}=0.6 \mathrm{~m} / \mathrm{s}$

It can be seen from FIG. 2, FIG. 3 and FIG. 4 that at the same inlet air temperature, the air outlet temperature increases significantly with the increase of air inlet velocity. This is because the speed increases, the contact time between the air and the fin heat exchange process becomes shorter, so that the air is unable to fully exchange heat with the fin in a short time and the outlet temperature rises. According to the velocity distribution diagram, the air velocity becomes very small in a small area at the rear of the tube with different inlet velocities, and the increase of inlet velocity increases the area with small velocities. This is because air swirls at the rear of the tube and the increase of velocity gradually expands the swirl area. The existence of swirl would not only increase the flow resistance and increase the power consumption, but also greatly weaken the heat transfer of the fin near the central region of the swirl, which is consistent with the rule of "prethinning and densification". In the latter half of the tube, the heat transfer between the air and the fin is greatly weakened and needs to be strengthened. Therefore, the opening at this point should be closer, which can be explained by the above calculation results. From the point of view of the whole flow field, the distribution of temperature field is greatly affected by the velocity field. Therefore, the appropriate inlet velocity has an important influence on the performance of heat exchanger. The average convection heat transfer 
coefficient and the pressure drop at the inlet and outlet of the fluid are two important indexes to evaluate the heat transfer performance of the heat exchanger. Therefore, the relation between the convection heat transfer coefficient of the heat exchanger and the pressure drop at the inlet and outlet with the wind speed is given in FIG. 5 and FIG. 6.

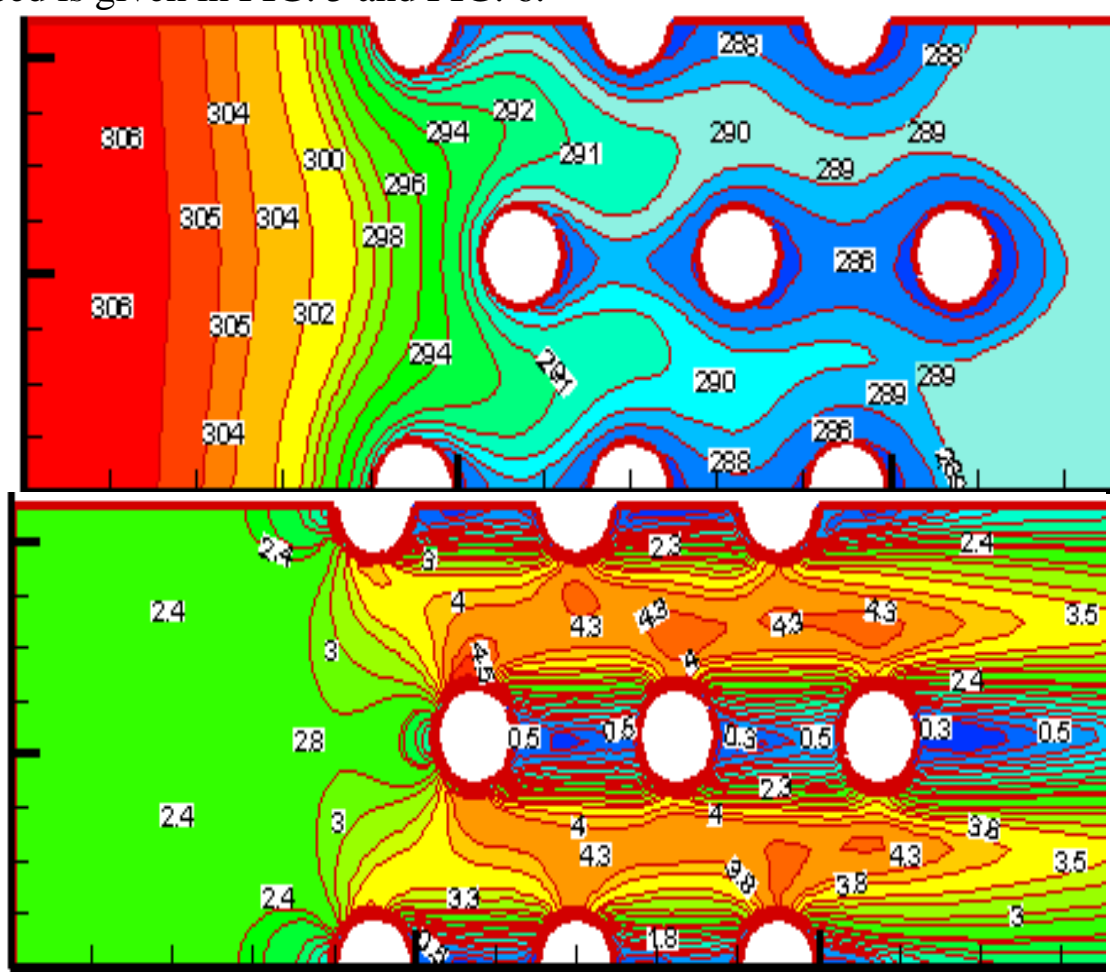

Fig. 3 cloud diagram of air temperature and velocity distribution when $V=2.4 \mathrm{~m} / \mathrm{s}$

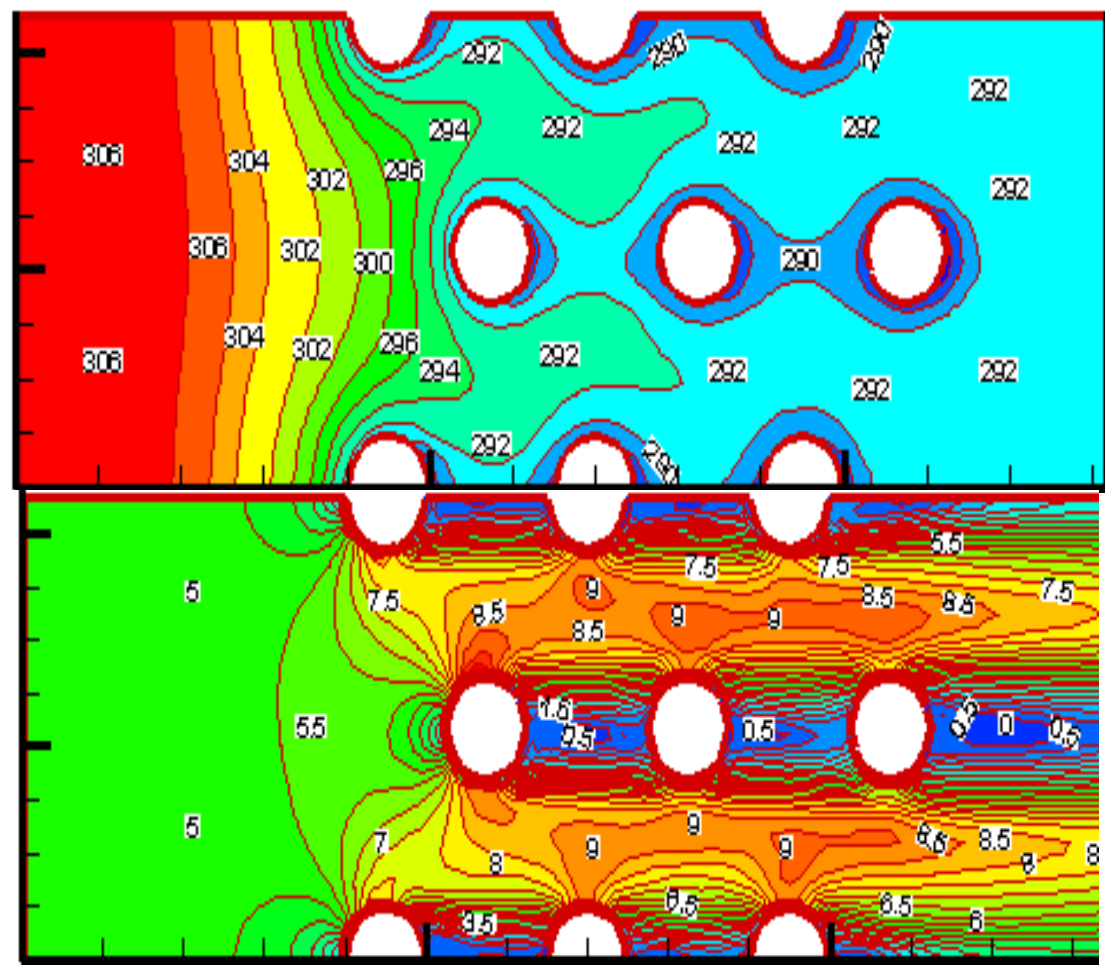

Fig. 4 cloud diagram of air temperature and velocity distribution when $\mathrm{V}=5.0 \mathrm{~m} / \mathrm{s}$ 


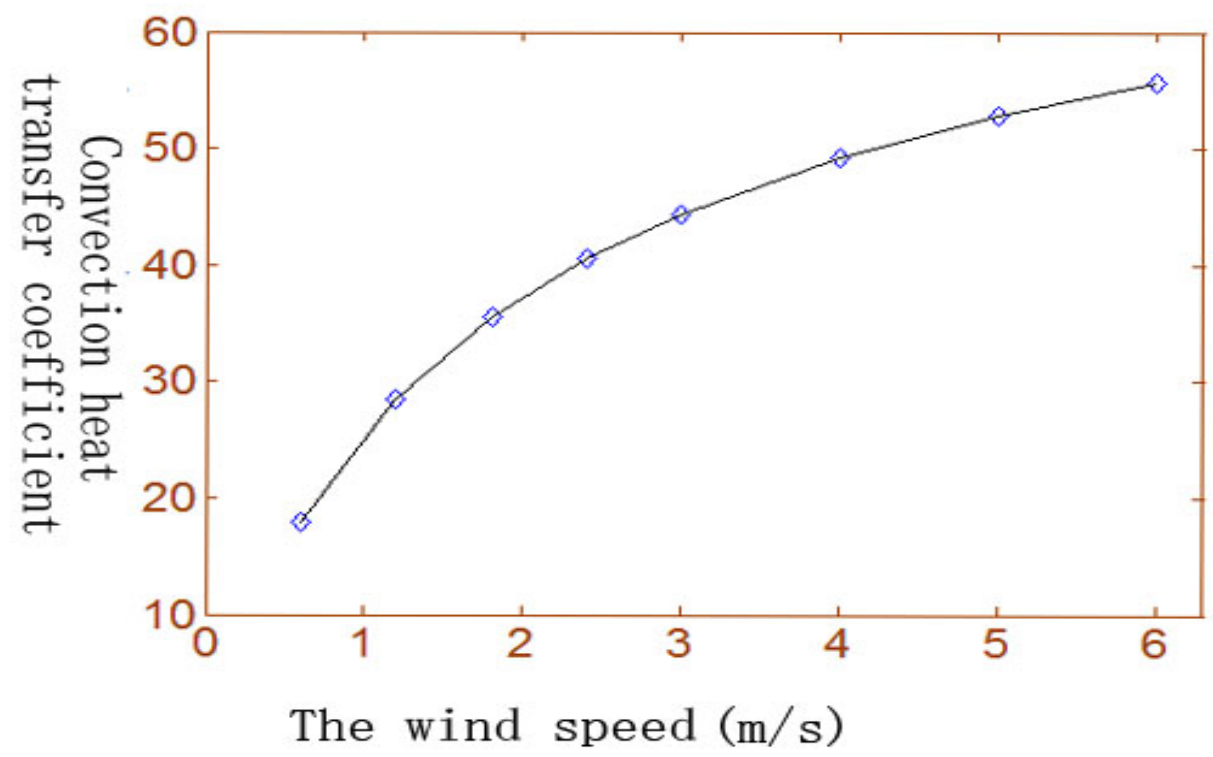

Fig. 5 relation between convective heat transfer coefficient and wind speed

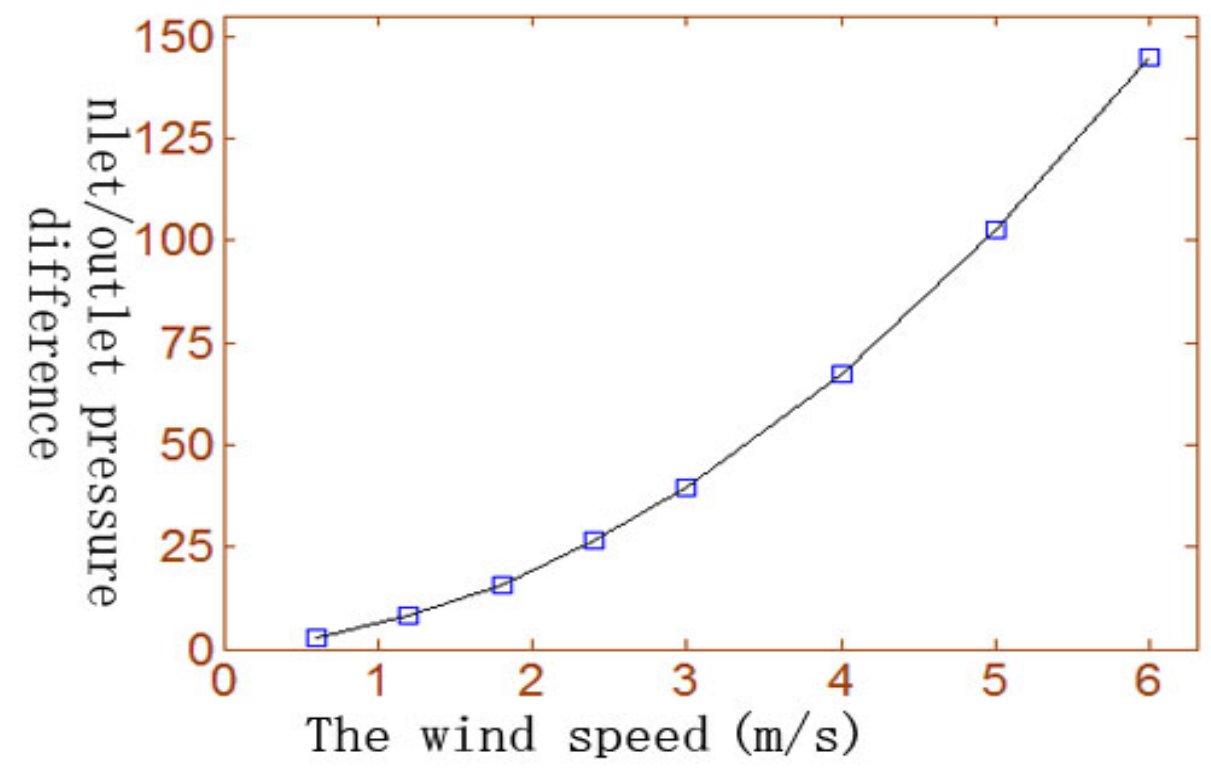

Fig. 6 relation of pressure difference at inlet and outlet with wind speed

It can be seen from figure 5 that the convective heat transfer coefficient of air and fin increases with the increase of wind speed, but the increasing rate decreases. FIG. 6 shows the relation between the pressure drop at the inlet and outlet and the wind speed. It can be seen that the increase of wind speed leads to the increase of pressure drop at the inlet and outlet of the air, and the increase rate is accelerated. Combined with figure 5 and 6 , it can be seen that the increase of wind speed brings a slow increase of convective heat transfer coefficient, but leads to a faster increase of pressure drop at the inlet and outlet of the air. In other words, within a certain wind speed range, the convective heat transfer coefficient changes significantly with the increase of wind speed. When the wind speed increases to a certain extent, the convective heat transfer coefficient increases slowly, but the pressure drop at the inlet and outlet of the air increases rapidly in this process. Therefore, for finned 
heat exchanger, the heat transfer coefficient of convection can be improved without increasing the wind speed. Meanwhile, the problem of increasing wind speed leads to the increase of resistance should be taken into account, which should be paid more attention to the design of heat exchanger.

\subsection{Field coordination analysis of heat transfer process}

The energy equation of boundary layer flow is integrated in the thermal boundary layer by Chinese scholars [3-6], which proves that reducing the Angle between velocity vector and temperature gradient is an effective measure to strengthen convective heat transfer. This basic idea is called the Field synergy principle. Later, this theory has been constantly verified and applied in numerical and experimental studies. Literature [7-8] has a detailed introduction, which will not be repeated here. In order to further analyze the basic mechanism of the convective heat transfer between air and fin, this process is analyzed by using the principle of field cooperation.

The mean value of Angle between velocity field and temperature field is defined as:

$$
\cos \beta=\frac{7}{4} \frac{h \mu}{4 \rho \lambda u_{m} p_{r}}
$$

type: $u_{m}$ is the minimum cross section average wind speed $(\mathrm{m} / \mathrm{s}) ; p_{r}=0.7, \mu$ is the dynamic viscosity $(\mathrm{Pa} / \mathrm{s})$.

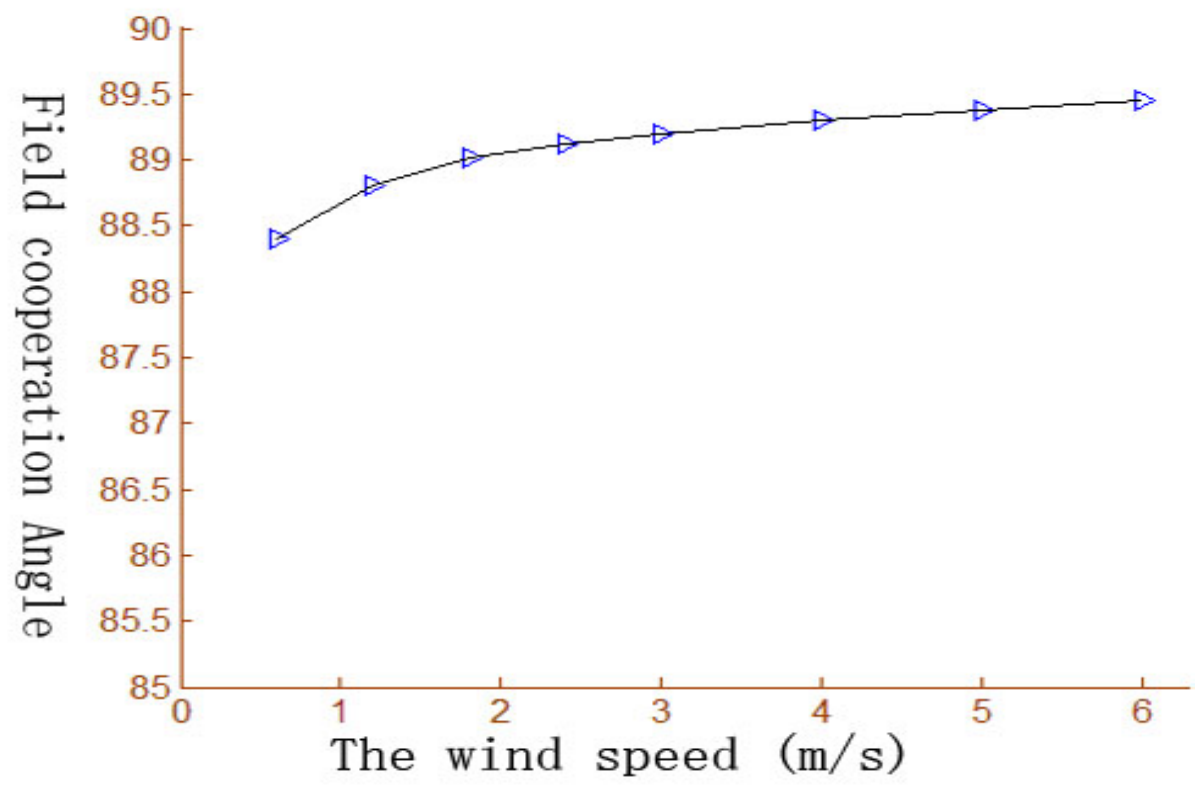

Fig. 7 relation of mean included Angle with wind speed

As can be seen from figure 7, with the increase of wind speed, the average field synergy Angle gradually increases. According to the principle of field synergy, the increase of wind speed leads to the poor synergistic performance of the velocity field and the temperature gradient field in the convective heat transfer process, which weakens the convective heat transfer process to some extent under the same conditions. And the increase of wind speed can indeed bring the increase of heat exchange, but the root cause of the increase of heat exchange in this process is the increase of the flow brought by the increase of wind speed. 


\section{Conclusion}

(1) when the air inlet temperature is constant, the increase of wind speed reduces the temperature difference between air inlet and outlet. The increase of wind speed leads to the increase of the back-swirl area of the tube and the increase of viscous resistance of the internal flow.

(2) the convective heat transfer coefficient of air and fin heat exchanger increases with the increase of wind speed, but the increasing rate decreases. The pressure drop of air inlet and outlet increases with the increase of wind speed.

(3) the root cause of the increase of heat transfer caused by the increase of wind speed is the increase of flow caused by the increase of wind speed; When the wind speed increases, the synergistic performance of temperature gradient field and velocity field becomes worse during convective heat transfer.

\section{References}

[1] Tao wenquan. Numerical heat transfer (2nd edition)[M]. Xi 'an: xi 'an jiaotong university press, 2001. Pp353-356.

[2] Guo zengyuan, Huang suyi. New technology of field coordination principle and enhanced heat transfer [M]. Beijing: China electric power press, 2004. P39 - 44.

[3] Guo da,Li Wang .Novel Concept for Convective Heat Transfer Enhancement[J].International Journal of Heat mass Transfer,1998,41(14):2221-2225.

[4] Wang shangdong. Novel concept and device of heat transfer augmentation[C].Proceedings of 11th International Conference of Heat Transfer, August 23-28, 1998, Kyongju, Korea. 1998, 5:405-408.

[5] GUO zhijie, S. Novel concept and approaches of heat transfer enhancement[C].Proceedings of Symposium on Energy Engineering in the 21st Century,New York:Begell House, 2000:118-126.

[6] Guo zhijie, Tao yuan. The field synergy (coordination) principle and its applications in enhancing single phase convective heat transfer [J].International journal of heat and mass transfer,2005,48(9):1797-1807.

[7] He yaling,Tao wenquan. Basic mechanism of strengthening single-phase convection heat transfer [J]. Journal of mechanical engineering, 2009.3 45(3):27 - 3. 